FACTA UNIVERSITATIS

Series: Mechanical Engineering Vol. 17, No 3, 2019, pp. 415 - 424

https://doi.org/10.22190/FUME181219003T

Original scientific paper

\title{
REDESIGN AND TOPOLOGY OPTIMIZATION OF AN INDUSTRIAL ROBOT LINK FOR ADDITIVE MANUFACTURING
}

\author{
Evangelos Ch. Tsirogiannis, George-Christopher Vosniakos
}

National Technical University of Athens, School of Mechanical Engineering, Section of Manufacturing Technology, Greece

\begin{abstract}
Design optimization for Additive Manufacturing is demonstrated by the example of an industrial robot link. The part is first redesigned so that its shape details are compatible with the requirements of the Selective Laser Sintering technique. Subsequently, the SIMP method of topology optimization is utilized on commercially available software in order to obtain the optimum design of the part with restrictions applicable to Additive Manufacturing, namely member thickness, symmetry and avoidance of cavities and undercuts. Mass and strain energy are the design responses. The volume was constrained by a fraction of the initial mass. The desired minimization of maximum strain energy is expressed as an objective function. A 7\% reduction in the mass of the part was achieved while its strength and stiffness remained unchanged. The process is supported by topology optimization software but it also involves some trial-and-error depending on the designer's experience.
\end{abstract}

Key Words: Topology Optimization, Robot Link, Lightweight, Design for Additive Manufacturing, Additive Manufacturing, Selective Laser Sintering

\section{INTRODUCTION}

In the last few years, topology optimization (TO) has emerged as a valuable tool for developing new design proposals within the framework of lightweight engineering, e.g. in the automotive industry [1], in the aircraft industry [2], but also in robotic mechanical structures, e.g. industrial [3], DLR [4] and humanoid robots [5]. A lightweight industrial robot implies safer close collaboration between human and robot in addition to energy efficiency, high positioning accuracy, payload capacity and lower requirements of the pertinent connection structure.

In topology optimization problems in real life, each finite element within the design domain is defined as a design variable, allowing a variation in density (homogenization,

Received December 19, 2018 / Accepted February 02, 2019

Corresponding author: George-Christopher Vosniakos

National Technical University of Athens, School of Mechanical Engineering, Section of Manufacturing

Technology, Heroon Polytechniou 9, 15780 Zografou, Athens, Greece

E-mail: vosniak@central.ntua.gr 
SIMP) [6] or void-solid representation (BESO) [7]. Additional well-known topology optimization methods are: homogenization [8], ground structure [9], the level-set [10] and the genetic method [11].

In the past, lightweight structures resulting from topology optimization were meant to be produced by material removal or other conventional manufacturing technologies, but, more recently, Additive Manufacturing (AM) methods are focused on [12, 13]. AM, a layer-wise material addition process family, may enable complex geometry and material distribution [14] with increases in strength and stiffness, and, at the same time, reduced weight of the part. Different reasons concerning fabrication time and cost minimization, including decentralized and on demand manufacturing, modifications and redesigns without penalties, possibility to make any complexity of geometry at no extra cost and time, increased supply chain proficiency as well as reduced environmental footprint are pointed out [14]. Certainly, the materials should comply with the design and manufacturing techniques. Printing of long fiber composites is not achievable yet but functional parts can be printed directly with metal powders [15]. Multiscale structures (foam, 2D / 3D lattice) [16] and multi-material design are also fostered by DFAM and can be used in combination with structural optimization [13, 17].

This work advocates the combination of TO and Design for Additive Manufacturing (DFAM) for developing lightweight industrial robot links. As an example, for a particular loading case, the forearm link of an existing robot is redesigned and topologically optimized for SLS. The principal selection criteria of the latter were total cost, accuracy and surface quality in comparison to other AM process capable of printing metal [14]. In Section 2 the principles of Design for AM are presented. In Section 3 redesign of the particular robot link addressed is outlined to conform to manufacturing by SLS. In Section 4 TO as applied in this case is presented and the pertinent results are discussed. Conclusions are summarized in Section 5.

\section{DESIGN FOR SLS: PRINCIPLES}

A collection of principles to be followed in applying design for SLS is presented next within the framework of lightweight engineering. Lightweight engineering for AM mainly refers to requirements, which are used for thickness distribution. It comprises: (a) design, referring to the creation of optimum geometries and shapes, (b) material, which addresses high stiffness-to-weight ratio and (c) manufacturing, which, in the current study, will take advantage of the SLS AM technology.

\subsection{DFAM Constraints}

Following specific design rules, robust geometry can be generated for SLS [18], an AM variant providing high accuracy and surface quality at affordable cost [14] as follows.

In SLS there is no need for support structures since the powder bed provides for it. As a result, overhangs may be blamed for lower heat conduction but not for the lack of support [12]. A negative or zero inclination angle between a layer and its previous layer denotes the lack of overhangs and minimal distortion of the part. An inclination angle between approximately $45^{\circ}$ and $90^{\circ}$ leads to larger but usually tolerable geometrical distortion of the part. For angles smaller than approximately $45^{\circ}$ not only is geometrical distortion large but surface quality of the overhanging structure is also low, the more so when 
inclination approaches $0^{\circ}$ [19]. Thus, building orientation should be chosen properly, to achieve an inclination angle larger than approximately $45^{\circ}$ or even better close to $90^{\circ}$ in most layers. If no building orientation gives acceptable results, part design may have to be modified. A similar approach applies to undercuts, as well.

Moreover, in SLS the minimum feature size should be respected. This is related to the minimum section size as constrained by the laser spot diameter. In addition, the bridging distance, i.e. the maximum physical gap that can be tolerated, should also be respected. In both cases suitable modifications in part design may be necessary [12].

The use of curves instead of corners on a layer profile could minimize high acceleration and deceleration stages of metal deposition which cause variations of the deposited material height [12].

Note that the design optimization for AM is affected by the lack of full specification of mechanical and thermal properties of the powder materials. In addition, anisotropy often results due to preferential crystal growing directions [20,21].

\subsection{Topology optimization for AM}

Having achieved a shape compatible with AM, topology optimization for AM follows. TO is usually applied to create lighter and stiffer structures by changing the material and the thickness distribution within the allowable limits dictated by the AM process. Thickness reduction as well as the creation of so-called 'multiscale' structures (e.g. foam, 2D and 3D lattice) results in lower residual stresses and lower distortion during and after the AM process. However, constraints and restrictions pertaining to AM should be taken into account.

Taking into account that TO is most commonly implemented on a Finite Element mesh, the main constraint is mesh resolution. A refined mesh implies the emergence of further detail and the improved topology. Furthermore, each section of the component should comprise at least 2-3 Finite Elements to obtain accurate calculation of the displacement, leading to a large number of design variables. Alleviating actions could be: hard-kill element elimination, iterative re-meshing and boundary-based TO methods [13]. After TO, complexity is high and the current methods cannot convert the model into a CAD file accurately. Hence, the model is usually converted into a Stereolithography (.stl) file.

Restrictions on TO apply to the design variables and may refer to (a) 'frozen' regions from which material cannot be removed, usually for reasons of interfacing of the part in an assembly, (b) the maximum and minimum member thickness, the former being due to functional reasons and the latter to AM process capability, (c) symmetry required for mass balancing purposes, and (d) avoidance of cavities (voids) or undercuts, due to the AM capabilities, too.

\section{REDESIGN FOR AM}

\subsection{Original design}

In the present study, the forearm of Stäubli RX90BL robot arm is dealt with. It is considered to be a high speed, low payload ( $4 \mathrm{~kg})$, high accuracy and repeatability $(50 \mu \mathrm{m})$ arm. The link was reverse-designed on CatiaTM V5 CAD system, see Fig. 1(a), from in-situ measurements. Several points can be noted with respect to the shape of the forearm link. It is monocoque for high stiffness, without holes or gaps to conform to protection class IP65. Since rotary joints generate linear accelerations increasing with the distance from the joint axis, a 
tapered cross section or wall thickness is presumed to reduce the associated inertial loads. Functional (mating) surfaces with the neighboring elbow and wrist links on either side of the forearm respectively, have high accuracy and surface finish.

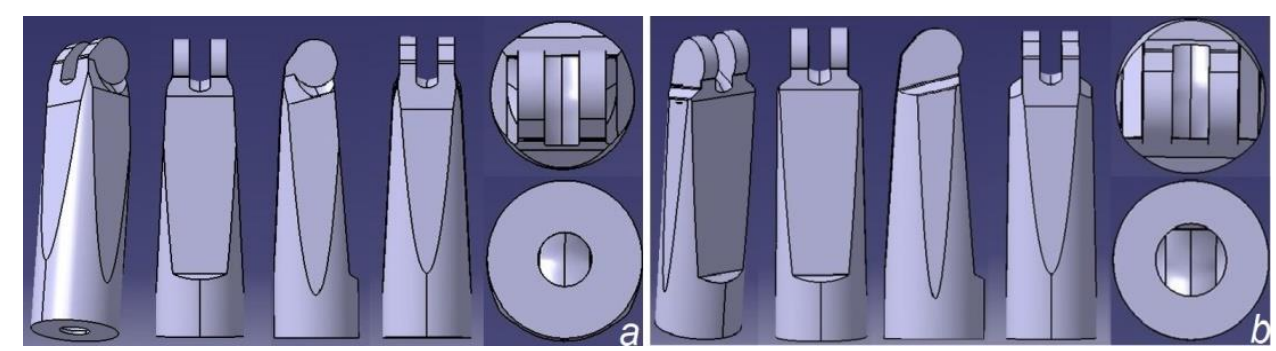

Fig. 1 Forearm (from left: isometric, front, left, back, top-bottom views) (a) original, (b) redesigned

\subsection{Modified design}

The forearm link (Fig. 1(a)) should be redesigned, at least locally, so that it can be produced by SLS process, see Fig. 1(b). The building direction is selected as parallel to the direction of its largest dimension as demonstrated in Fig. 2(a).
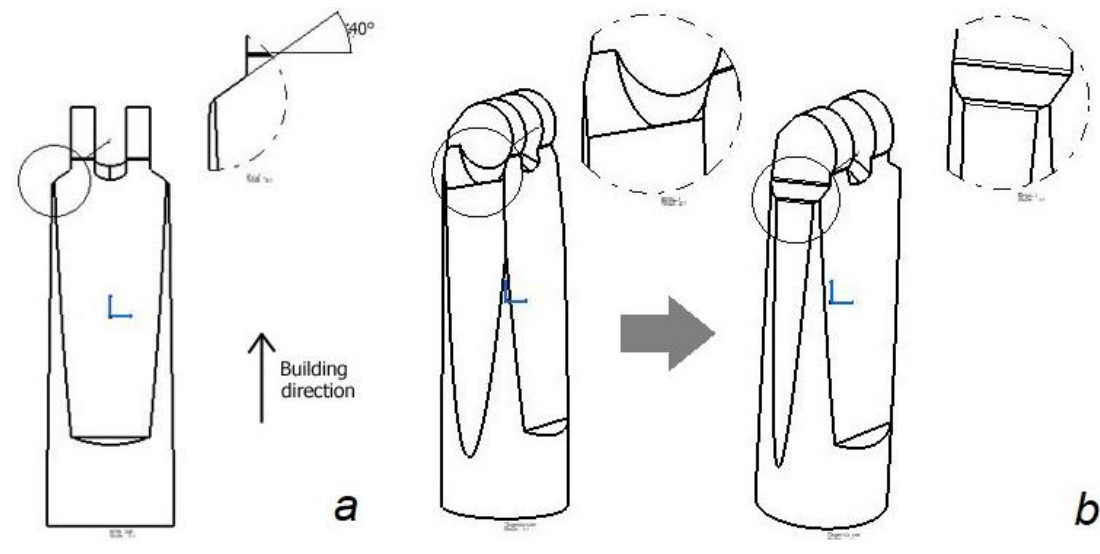

Fig. 2 Redesign for SLS details (a) inclination angle (b) modification of the upper end

Based on that, the inclination angle at its upper end is selected as $40^{\circ}$ from the horizontal (Fig. 2(a)) in order to obtain acceptable overhangs without support. Furthermore, intricate features have been replaced by simpler ones. For example, some corners are replaced by particular curves (Fig. 2(b)) to minimize overhanging structures. Note that in this case overhangs are formed in the interior of the link and not on its external shape. Simplified geometry is expected to yield better dimensional accuracy and surface quality in SLS production as well as lower stress concentration. 


\subsection{Material selection}

Available alloys used in the SLS process include 17-4 and 15-5 stainless steel, maraging steel, cobalt chromium, inconel 625 and 718, aluminium AlSi10Mg, and titanium Ti6Al4V [22]. AlSi10Mg alloy is selected since it is often used for products with thin walls and complex shapes, exhibiting good strength, strength to weight ratio, toughness, dynamic properties and recyclability [21]. Mechanical properties of the material are selected equal to those in $\mathrm{z}$ (building) direction since they represent the worst case scenario of isotropic material being inferior to the properties along $\mathrm{x}$ and $\mathrm{y}$ directions. The properties of interest are presented in Table 1. Safety factor for the AlSi10Mg alloy on the basis of yield strength is selected at 1.25 [23]. Thus, the forearm mass is calculated at $5.717 \mathrm{~kg}$.

Table 1 Properties of AlSi10Mg alloy [22]

\begin{tabular}{lll}
\hline Property & Value & Unit \\
\hline Young modulus & 62000 & $\mathrm{MPa}$ \\
Yield strength & 230 & $\mathrm{MPa}$ \\
Density & 2.68 & $\mathrm{gr} / \mathrm{mm}^{3}$ \\
\hline
\end{tabular}

\subsection{Analysis}

The static analysis of the forearm structure was performed in Abaqus ${ }^{\mathrm{TM}}$, in order to calculate reference stress and strain distribution to which the distribution that is expected to result from TO should be compared. Three partitions are created by two planes, see Fig. 3(a), for generating different meshes as necessary as well as for determining the design area in TO.

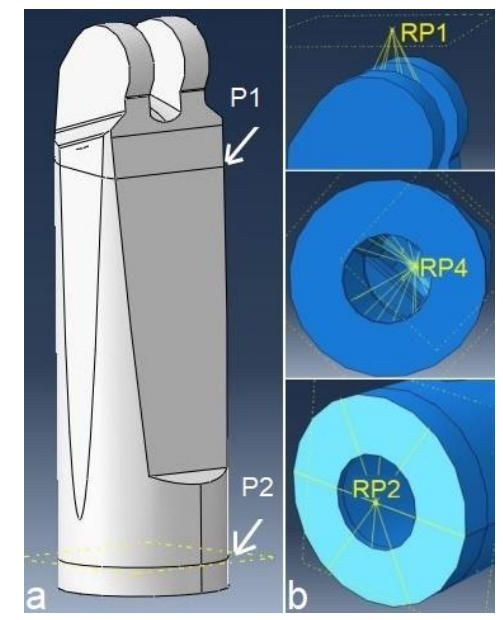

Fig. 3 (a) Partitions P1-P2 (b) Load application points (RP1: wrist and tool center of gravity, RP4: forearm center of gravity, RP2: elbow joint torque center)

The loads on the robotic forearm are estimated for a random position of the robot arm movement. For the sake of brevity, the center of gravity between the tool (a 3D printer head in this case), the end effector and the wrist link were calculated and the corresponding reference point RP1, which represents the center of gravity, is defined as shown in Fig. 3(b). Similarly, 
another two reference points are also defined, i.e. RP4, representing the center of gravity of the forearm, and RP2 located on the bottom flange of the forearm, where the torque of the elbow joint motor is applied, see Fig. 3(b). Continuum distributing couplings (as defined in Abaqus ${ }^{\mathrm{TM}}$ ) were used to connect these points with the pertinent areas of the forearm. The loads applied are shown in Table 2.

A moment resulting by the motor of the wrist joint is also applied. The magnitude of that moment is $57 \mathrm{Nm}$. Accordingly, a boundary condition is applied for 5 Dofs (Fx, Fy, $\mathrm{Fz}, \mathrm{My}, \mathrm{Mz}$ ) on the bottom of the forearm.

Table 2 Loads applied

\begin{tabular}{|c|c|c|c|c|c|c|}
\hline & $F_{x}(N)$ & $\mathrm{F}_{\mathrm{y}}(\mathrm{N})$ & $\mathrm{F}_{\mathrm{z}}(\mathrm{N})$ & $M_{x}(\mathrm{Nm})$ & $\mathrm{M}_{\mathrm{y}}(\mathrm{Nm}$ & $\mathrm{M}_{\mathrm{z}}(\mathrm{Nm})$ \\
\hline RP1 & 300 & 250 & 50 & 200 & 175 & 350 \\
\hline RP4 & 700 & 500 & 100 & 300 & 250 & 1000 \\
\hline RP2 & & & & 100 & & \\
\hline
\end{tabular}

Abaqus ${ }^{\mathrm{TM}} \mathrm{C} 3 \mathrm{D} 10$ (10-node quadratic tetrahedron) element type was used in meshing. The density of the mesh is specified as 5 by creating seeds along the edges of the model. The free meshing technique is selected as it is the most flexible top-down methodology fully associated with the geometry of the model.

During the linear static analysis, it was noticed that the use of connections for pre-process modeling of the loads increased memory allocation on a 8 GB RAM, Intel i7 $2.90 \mathrm{GHz}$ computer, eventually making it impossible to proceed to the solution. Hence, as few connections as possible were used. Afterwards, the connections were divided into smaller subconnections by selecting the pertinent sub-surfaces for each sub-connection, ultimately decreasing memory allocation to $7973 \mathrm{MB}$. Approximately 30 attempts were needed with a mean duration of 15 minutes each in order to solve the problem in a trial and error fashion.

The results obtained concerning von Mises stresses and deformations are shown in Fig. 4. The results present a maximum stress value of $10.73 \mathrm{MPa}$ and deformations up to $0.09 \mathrm{~mm}$, both being considered low and, thus, acceptable.

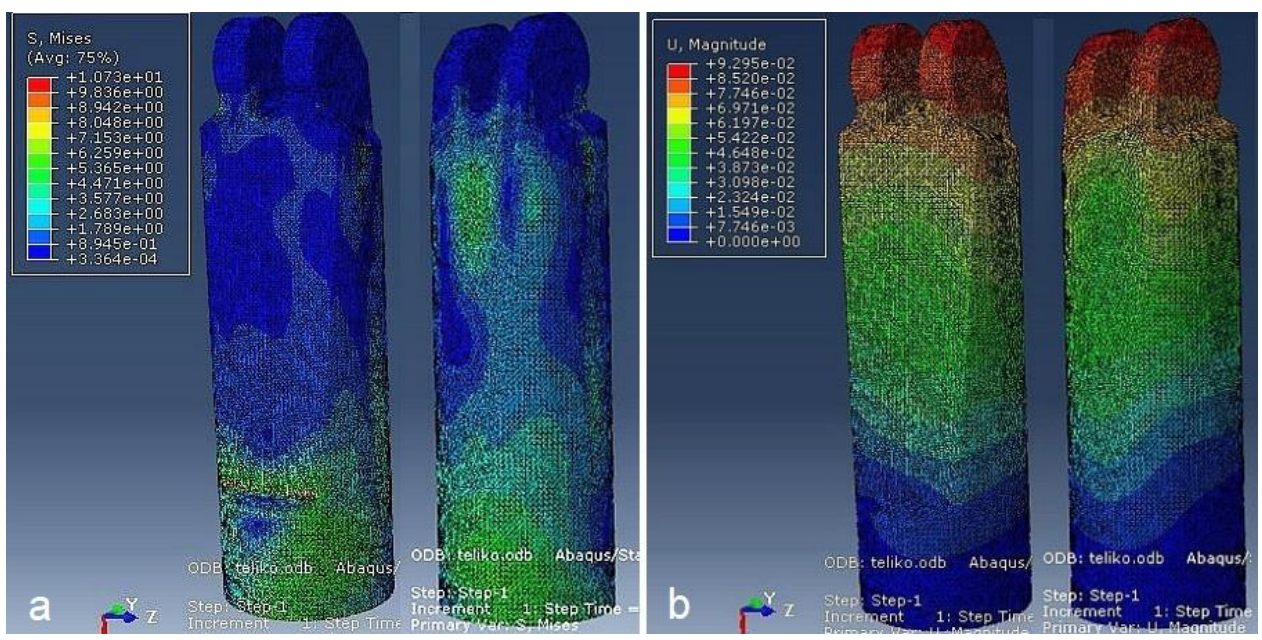

Fig. 4 Analysis results before TO (a) von Mises equivalent stress (b) Deformation 


\section{TOPOLOGY OPTIMIZATION FOR AM}

SIMP (Solid Isotropic Material with Penalization) TO method was used due to its reliability, efficiency and ease of setup, including low storage space and computational load [24].

\subsection{Setup}

Initially, the model was partitioned by two planes P1 and P2, see Fig. 3(a) between which the design area is defined, whereas the rest had to stay unchangeable.

The design variables are the densities of the elements in the design area, which take discrete $0-1$ values. Two design responses were considered, namely strain energy and volume. The objective function was specified so as to minimize the maximum strain energy calculated for all the elements. The limit applied to the value of volume response is specified as a maximum reduction by $7 \%$ from its initial value. Consequently, the mass of the forearm's design section would also be reduced by a maximum of $7 \%$.

Next, restrictions are taken into consideration. Since TO in Abaqus ${ }^{\mathrm{TM}}$ does not support AM-specific restrictions, the most akin ones are selected. These include: (a) restrictions on cavities and undercuts (b) minimum member thickness at $1 \mathrm{~mm}$, equal to the laser beam diameter [13] (c) planar symmetry between the right and the left side of the part for the forearm to comply with the design rules of robot arms.

The global stop condition was obtained by trial and error at 22 design cycles. The general TO algorithm applied adjusts density and stiffness while trying to satisfy the objective function and the constraints as outlined in detail in [25]. The same computer and memory allocation as those described in Section 3.4 were used in TO, too.

\subsection{Procedure}

Next, the steps leading to the optimization results are explained and shown diagrammatically in Fig. 5.

In the first phase, attempts were made to achieve a modified forearm design with reduced mass retaining in parallel the strength and stiffness characteristics of the initial design. The objective function to minimize the strain values and the solid volume/mass constraint are defined by the user. Thus, after repeated trials, each lasting about $15 \mathrm{~min}$, it was noticed that for mass reduction higher than $10 \%$ the optimization problem could not be solved.

In the second phase, apart from the mass, strength and stiffness considerations the symmetry and member thickness restrictions, see Section 4.1 , were introduced with objective function as: "minimize strain energy values". Five runs were needed to ascertain that the manufacturing restrictions, in particular the minimum thickness, could not be satisfied with $10 \%$ mass reduction, leading to a lower figure of $7 \%$.

In the third phase, the target of the objective function was set as to "minimize the maximum strain energy values", the rest of the setting being kept as in the second phase. The resulting robot arm geometry was better compared to the second phase, due to the more homogenous spread of thickness reduction. Note that subsequent execution of the optimization for $8 \%$ mass reduction was not successful due to the minimum thickness restriction and, as a result, the highest mass reduction was again $7 \%$.

In the fourth phase, the restriction on cavities and undercuts was added, see Section 4.1. The resulting geometry did not have cavities and undercuts anymore and, as a result, it was better in terms of manufacturability compared to the third phase. Again, minimum thickness restriction impaired increase of mass reduction to $8 \%$. 

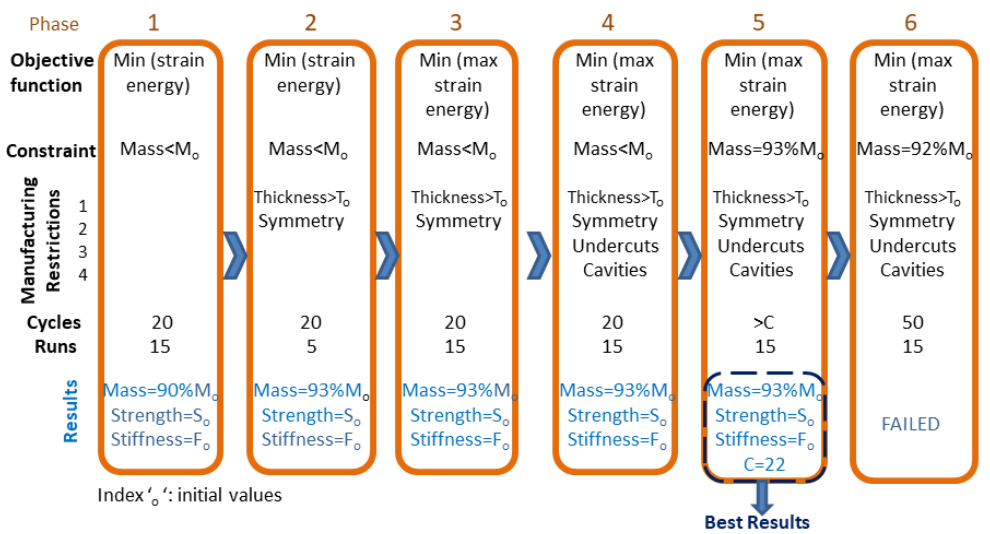

Fig. 5 Topology optimization procedure

The first four phases amounted to about 50 runs, each lasting about $7 \mathrm{hrs,} \mathrm{the} \mathrm{optimization}$ maximum number of cycles being defined as 20 . The latter, definitely affect quality of optimization results, thus the optimum number of cycles was further explored in the fifth phase. It was observed that the best results were obtained with 22 cycles, with duration of approximately $8 \mathrm{hrs}$. In the sixth phase, an attempt to reduce the forearm mass by $8 \%$ by increasing the maximum number of cycles to 50 was not successful.

\subsection{Results}

After progressive removal of the necessary elements to create voids in the internal and the external shape of the forearm, the modified forearm was obtained, see Fig. 6.
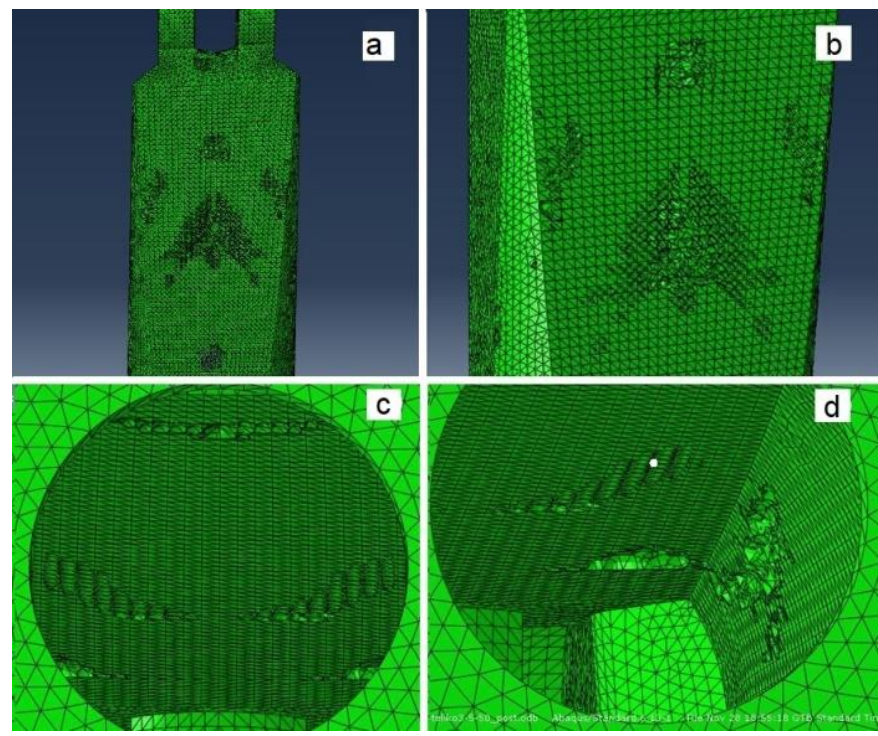

Fig. 6 Removal of elements: (a) and (b) external shape (c) and (d) internal shape 
Equivalent von Mises stresses and deformations of the forearm after TO are shown in Fig. 7. The results present a maximum stress value of $10.72 \mathrm{MPa}$ and deformations up to $0.09 \mathrm{~mm}$. Based on mass, stress and deformation comparison, the topologically optimized link is better than the original one: its mass is reduced by $7 \%$, it sustains the same mechanical load while respecting the same maximal displacement and maximal Von Mises stress, which is obvious by comparing Fig. 4 and Fig. 7.
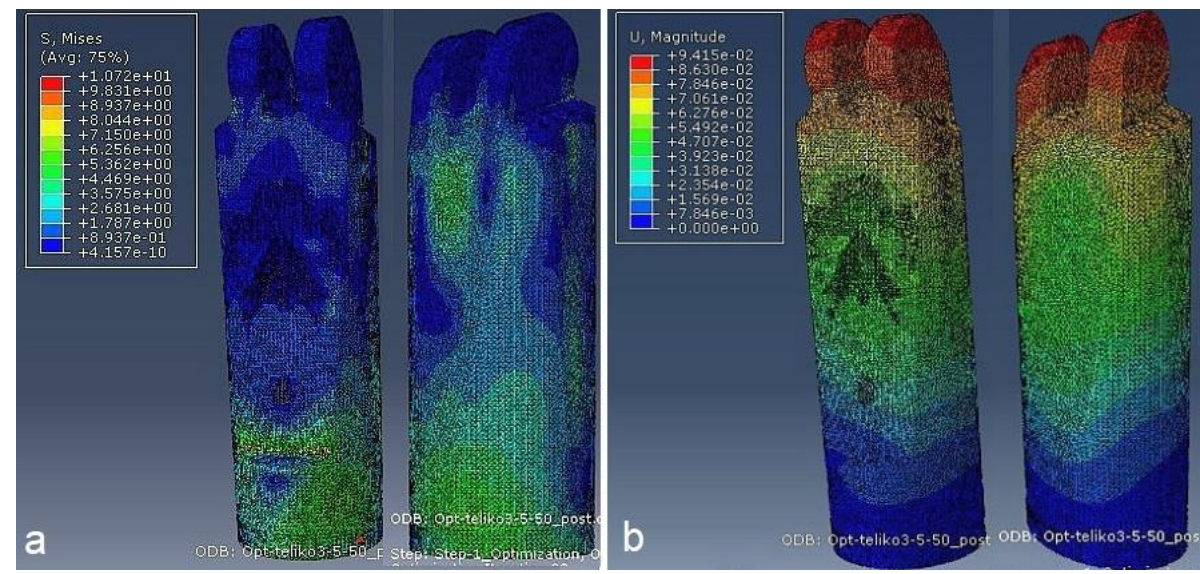

Fig. 7 Results after TO: (a) von Mises equivalent stress (b) Deformation

\section{CONCLUSION AND PERSPECTIVES}

This study presents an overall structural design optimization approach for a robot arm link seeking mass reduction and satisfaction of manufacturability with SLS AM technique. Mass reduction achieved is deemed moderate at $7 \%$ but it is worth noting that the maximum deformation and the maximum von-Mises stresses of the customized link have remained the same.

At the early design stage, FEA and TO simulations are very effective as the designer can consider the capabilities of AM and obtain the optimal geometry in terms of stress, deformation and weight response. Still, TO is a trial-and-error process, depending to some extent on designer experience in problem modeling. However, TO does restrict extensive trial-and-error, which would otherwise be necessary at a much higher level of part design until the final shape is reached.

The use of AM and DFAM methods is expected to increase substantially in the industrial sector in the years to come. Thus, robot industrialists could benefit from short lead times, fast iteration cycles and low costs as well as the TO capabilities of AM. Nevertheless, TO is currently a time-intensive task, which can be significantly improved by multiple core processors or special hardware for accelerating computations.

As future work, it would be most advantageous to extend TO towards rigid body dynamics in order to consider all the possible robot poses and not only one, thus incorporating a multitude of loading cases. In addition, TO could be followed by shape Optimization in order to obtain smoother geometries. Last but not least, further development of the existing TO software programs would be useful in order to adapt to AM technologies, introducing AM specific restrictions. 


\section{REFERENCES}

1. Raugei M., Morrey D., Hutchinson A., Winfield P., 2015, A coherent life cycle assessment of a range of lightweighting strategies for compact vehicles, J Clean Prod, 108, pp. 1168-1176.

2. Saleem W., Yuqing F., Yunqiao W., 2008, Application of Topology Optimization and Manufacturing Simulations - A new trend in design of Aircraft components, Proc. International Multi-Conference of Engineers and Computer Scientists. Hong Kong, p 6.

3. Yin H., Huang S., He M., Li J., 2016, An overall structure optimization for a light-weight robotic arm, IEEE 11th Conference on Industrial Electronics and Applications (ICIEA). pp 1765-1770.

4. Albu-Schäeffer A., Haddadin S., Ott C., Stemmer A., Wimbock T., Hirzinger G., 2007, The DLR Lightweight Robot - Design and Control Concepts for Robots in Human Environments, Ind Rob, 34, pp. 376-385.

5. Albers A., Ottnad J., 2009, Integrated structural and controller optimization for lightweight robot design, 9th IEEE-RAS International Conference on Humanoid Robots. pp. 93-98

6. Rozvany G.I.N., 2001, A critical review of established methods of structural topology optimization, Struct Multidiscip Optim, 37, pp. 217-237.

7. Young V., Querin O., Steven G., Xie Y., 1999, 3D and multiple load case bi-directional evolutionary structural optimization (BESO), Struct Optim, 18, pp.183-192.

8. Bendsoe M.P., Kikuchi N., 1988, Generating optimal topologies in structural design using a homogenization method, Comput Methods Appl Mech Eng, 71, pp. 197-224.

9. Dorn W.S., Gomory R.E., Greenberg H.J., 1964, Automatic design of optimal structures, J Mec, 3, pp. 25-52

10. Allaire G., Jouve F., Toader A.-M., 2002, A level-set method for shape optimization, Comptes Rendus Math, 334, pp. 1125-1130.

11. Wang S.Y., Tai K., 2005, Structural topology design optimization using genetic algorithms with a bitarray representation, Comput Methods Appl Mech Eng, 194, pp. 3749-3770.

12. Leary M., Merli L., Torti F., Mazur M., Brandt M., 2014, Optimal topology for additive manufacture: A method for enabling additive manufacture of support-free optimal structures, Mater Des, 63, pp. 678-690.

13. Brackett D., Ashcroft I., Hague R., 2011, Topology optimization for additive manufacturing, Solid freeform fabrication symposium. Austin, Texas, pp. 348-362.

14. Bikas H., Stavropoulos P., Chryssolouris G., 2016, Additive manufacturing methods and modeling approaches: A critical review, Int J Adv Manuf Technol, 83, pp. 389-405.

15. Hällgren S., Pejryd L., Ekengren J., 2016, (Re)Design for Additive Manufacturing, 26th CIRP Design Conference, pp 246-251.

16. Tang Y., Kurtz A., Zhao Y.F., 2015, Bidirectional Evolutionary Structural Optimization (BESO) based design method for lattice structure to be fabricated by additive manufacturing, Comput Des, 69, pp. 91-101.

17. Crescenzio F. De, Lucchi F., 2017, Design for Additive Manufacturing of a non-assembly robotic mechanism, Advances on Mechanics, Design Engineering and Manufacturing, Springer International Publishing.

18. Chahine G., Smith P., Kovacevic R., 2010, Application of Topology Optimization in Modern Additive Manufacturing, Solid Freeform Fabrication Symposium, Austin, Texas, pp. 606-618.

19. Goutianos S., 2017, Selective Laser Melting of Hot Gas Turbine Components: Materials, Design and Manufacturing Aspects, IOP Conf. Ser.: Mater. Sci. Eng., 219 012022, pp 1-8.

20. Vaneker T.H., 2017, The role of Design for Additive Manufacturing in the successful economical introduction of $A M, 27$ th CIRP Design Conference, pp. 181-186.

21. Salonitis K., Zarban S. Al, 2015, Redesign Optimization for Manufacturing Using Additive Layer Techniques, CIRP 25th Design Conference Innovative Product Creation Redesign, Elsevier B.V., pp. 193-198.

22. 3D Systems, 2018, Technical Specifications of Materials for Additive Manufacturing, www.3dsystems.com /materials (last access: 15.12.2018)

23. Maleque M.A., Salit M.S., 2013, Materials Selection and Design, Springer, Singapore.

24. Tang Y., Zhao Y., 2016, A survey of the design methods for additive manufacturing to improve functional performance, Rapid Prototyp J, 22, pp. 569-590.

25. Bendsoe M.P., Sigmund O., 2003, Topology Optimization, Springer. 